\title{
Estrategias para promover el acceso a medicamentos de interés en salud pública: revisión estructurada de la literatura
}

\author{
Strategies to promote access to medicines of interest in public health: structured \\ review of the literature
}

\author{
Estratégias para promover o acesso a medicamentos de interesse em saúde \\ pública: revisão estruturada da literatura
}

Mónica Ledezma-Morales', Pedro Amariles², Claudia Marcela Vargas-Peláez³, Francisco Augusto Rossi Buenaventura4.

1 Magíster en Investigación y Uso Racional del Medicamento, química farmacéutica, Universidad de Antioquia, Colombia. monica. ledezma@udea.edu.co, oRCID: https://orcid.org/0000-0003-4307-1282.

2 Doctor en Farmacia, químico farmacéutico, Universidad de Antioquia, Colombia. pedro.amariles@udea.edu.co, oRciD: https://orcid. org/0000-0002-3825-8045.

3 Doctora en Farmacia, química farmacéutica, Fundación IFARMA, Colombia. cvargas@ifarma.org, oRCID: https://orcid.org/0000-00015011-8718.

4 Epidemiólogo, médico, Fundación IFARMA, Colombia. frossi@ifarma.org, oRCiD: https://orcid.org/0000-0002-6172-5852.

Recibido: 15/05/2018. Aprobado: 18/12/2019. Publicado: 05/02/2020

Ledezma-Morales M, Amariles P, Vargas-Peláez CM, Rossi Buenaventura FA. Estrategias para promover el acceso a medicamentos de interés en salud pública: revisión estructurada de la literatura. Rev. Fac. Nal. Salud Pública. 2020;38(1):e332273. Dor: https:// doi.org/10.17533/udea.rfnsp.v38n1e332273

\section{Resumen}

Objetivo: Identificar estrategias orientadas a favorecer el acceso a medicamentos de interés en salud pública, de alto costo o protegidos por patentes, posiblemente aplicables al contexto colombiano. Metodología: revisión estructurada en PubMed/Medline, utilizando los términos MeSH: "health services accessibility", "pharmaceutical preparations", "policy", con filtros para artículos publicados en inglés y español, entre 2012 y 2017. Se incluyeron aquellos con información sobre estrategias o políticas que favorecieran el acceso a medicamentos de interés en salud pública de alto costo o protegidos por patentes. Las estrategias identificadas fueron agrupadas acorde con las cinco dimensiones del acceso a la atención en salud y medicamentos definidas por la Organización Mundial de la Salud: disponibilidad, asequibilidad, accesibilidad, aceptabilidad y calidad. Resultados: Se identificaron 62 artículos, de los cuales se incluyeron 18 y se consideraron 5 referencias adicionales. Se reconocieron 30 estrategias, distribuidas y clasificadas según las dimensiones del acceso: disponibilidad y asequibilidad, 15; accesibilidad, 6; aceptabilidad, 6; calidad, 2; estrategia transversal, 1. Conclusiones: Se identificaron estrategias claves para favorecer el acceso a medicamentos de interés en salud pública de alto costo o protegidos por patentes, dentro de las que se encuentran las negociaciones centralizadas de precios; el apoyo financiero público para la investigación, desarrollo y producción nacional de medicamentos genéricos; la aplicación 
de las flexibilidades del acuerdo sobre los "Aspectos de los derechos de propiedad intelectual relacionados con el comercio" y la implementación de programas de asistencia.
-Palabras clave: Accesibilidad a los servicios de salud; política de la salud; medicamento; propiedad intelectual; precio de medicamento.

\section{Abstract}

Objective: To identify strategies aimed at promoting access to high-cost or patent-protected medicines of interest in public health, possibly applicable to the Colombian context. Methodology: structured review in PubMed/Medline, using the MeSH terms: "health services accessibility", "pharmaceutical preparations", "policy", with filters for articles published in English and Spanish, between 2012 and 2017. Studies with information on strategies or policies that favored access to highcost or patent-protected drugs of interest in public health were included. The strategies identified were grouped according to the five dimensions of access to health care and medicines defined by the World Health Organization: availability, affordability, accessibility, acceptability and quality. Results: 62 articles were identified, of which 18 were included, and 5 additional references were considered. 30 strategies were recognised, distributed and classified according to the dimensions of access: availability and affordability, 15; accessibility, 6; acceptability, 6; quality, 2; cross-cutting strategy, 1. Conclusions: Key strategies were identified to favor access to high-cost or patentprotected medicines of interest in public health, including centralized price negotiations; public financial support for national research, development and production of generic medicines; the application of the flexibilities of the agreement on "Aspects of intellectual property rights related to trade" and the implementation of care programs.

--------Key words: Accessibility to health services; health policy; medicine; intellectual property; medicine price.

\section{Resumo}

Objetivo: Identificar estratégias destinadas a favorecer o acesso a medicamentos de interesse em saúde pública, de alto custo ou protegidos por patentes, possivelmente aplicáveis ao contexto colombiano. Metodologia: revisão estruturada no PubMed/Medline, usando os termos MeSH: "health services accessibility", "pharmaceutical preparations", "policy", com filtros para artigos publicados em inglês e espanhol, entre 2012 e 2017. Foram incluídos aqueles com informações sobre estratégias ou políticas que favoreciam o acesso a medicamentos de interesse em saúde pública de alto custo ou protegidos por patentes. As estratégias identificadas foram agrupadas de acordo com as cinco dimensões de acesso aos cuidados de saúde e medicamentos definidas pela Organização Mundial da Saúde: disponibilidade, alcançabilidade, acessibilidade, aceitabilidade e qualidade. Resultados: Foram identificados 62 artigos, dos quais 18 foram incluídos e 5 referências adicionais foram consideradas. 30 estratégias foram identificadas, distribuídas e classificadas de acordo com as dimensões do acesso: disponibilidade e alcançabilidade, 15; acessibilidade, 6; aceitabilidade, 6; qualidade 2; estratégia transversal, 1. Conclusões: Foram identificadas estratégiaschave para favorecer o acesso a medicamentos de interesse em saúde pública de alto custo ou protegidos por patentes, dentro das quais estão as negociações centralizadas de preços; o apoio financeiro público à pesquisa, desenvolvimento e produção nacional de medicamentos genéricos; a aplicação das flexibilidades do acordo sobre "Aspectos dos direitos de propriedade intelectual relacionados ao comércio" e a implementação de programas de assistência.

--------Palavras-chave: Acessibilidade aos serviços de saúde; política de saúde; medicamento; propriedade intelectual; preço do medicamento.

\section{Introducción}

En 1975, la 28. ${ }^{\text {a }}$ Asamblea Mundial de la Salud aprobó el concepto de medicamentos esenciales (ME), surgido de la necesidad de los Estados miembros de la Organización Mundial de la Salud (OMs) de contar con "asesoramiento sobre selección y adquisición, a precios asequibles, de medicamentos de calidad probada, indispensables para atender las necesidades nacionales de salud" [1]. Posteriormente, con la publicación del primer Listado de ME en 1977, comenzó la inclusión de los ME en los listados nacionales de medicamentos y la implementación de políticas farmacéuticas a nivel mundial [2].

A pesar del posicionamiento del concepto de ME y de que se han establecido medidas — como la creación 
de planes de beneficios en salud (PBS) — ${ }^{*}$ para garantizar el acceso equitativo a los tratamientos y controlar el gasto farmacéutico, algunos países de América Latina y del mundo mantienen el reto de lograr el acceso a los medicamentos como componente esencial del derecho a la salud, incluyendo a los ME, especialmente en las poblaciones pobres y vulnerables [2-4]. Datos de la oms sobre el acceso a ME indican que, durante el período 2007-2014, la disponibilidad promedio de ME fue solo del $60 \%$ en el sector público de países de bajos ingresos, y del $56 \%$ en países de ingresos bajos-medianos [5]. Adicionalmente, se estima que un tercio de la población mundial no tiene acceso a ME (y menos de la mitad en algunas áreas) [6], estando privados de tratamientos necesarios contra aquellas enfermedades que tienen un impacto negativo en su salud y bienestar.

Cabe señalar que las limitaciones al acceso a los ME en las enfermedades de interés en salud pública (transmisibles como el virus de inmunodeficiencia humana - VIH - o la hepatitis $\mathrm{C}$; y no transmisibles como la diabetes o la enfermedad pulmonar obstructiva crónica) pueden tener efectos deletereos en la población, debido a su prevalencia y al riesgo de aparición de otras enfermedades, secuelas definitivas, discapacidad o muerte. Adicionalmente, en enfermedades transmisibles como la tuberculosis, la hepatitis C y el VIH/Sida, la falta de continuidad en las terapias por dificultades en el acceso a los medicamentos puede favorecer la resistencia al tratamiento farmacológico o progresión de la enfermedad, lo que se asocia a un aumento en transmisibilidad y efectos negativos poblacionales [7].

Dos de los factores que han generado condiciones de inequidad y limitaciones de acceso a medicamentos han sido el modelo privatizado de innovación farmacéutica y la condición de monopolio que se ha instaurado con el otorgamiento de derechos de propiedad intelectual, que han permitido el establecimiento de altos precios en los medicamentos [4], especialmente a ME de interés en salud pública, como los antirretrovirales (ARV), antivirales de acción directa (AAD) para la infección por el virus de la hepatitis $\mathrm{C}$ (VHC), antineoplásicos, inmunomoduladores, entre otros.

En general, la oms establece que los países deberían formular e implementar políticas farmacéuticas para favorecer el acceso a ME, las cuales debería incluir estrategias/acciones en cinco dimensiones: disponibilidad, asequibilidad, accesibilidad, aceptabilidad y calidad [8]. Estas dimensiones pueden incorporarse a las políticas, independientemente del enfoque que adopten: 1) acceso, uso racional y calidad de los medicamentos; 2) cadena del medicamento (Investigación y Desarrollo
- $\mathrm{I}+\mathrm{D}-$, fabricación, autorización, distribución, comercialización, prescripción, dispensación, administración/ uso, disposición final) o 3) derecho a la salud como un derecho humano fundamental [9], puesto que presentan relaciones complementarias entre ellas (véase figura 1).

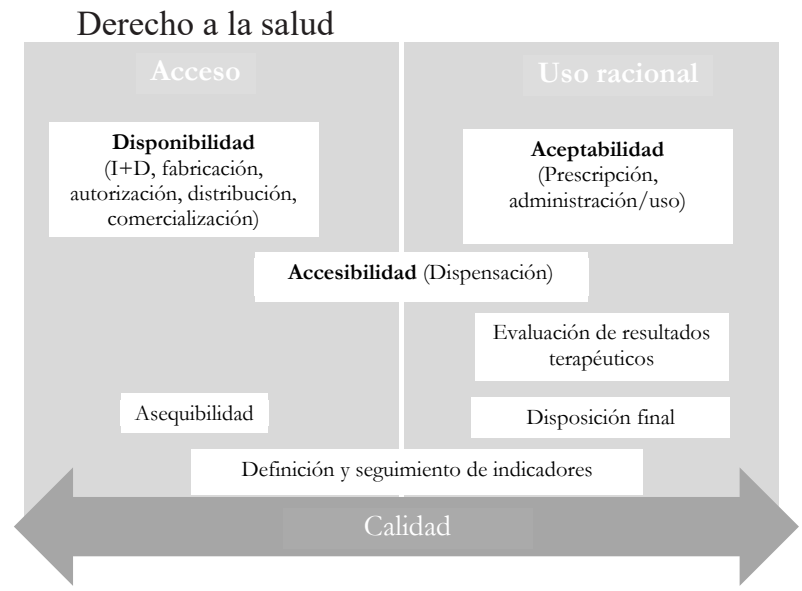

Figura 1. Relación entre las dimensiones del acceso a medicamentos y el enfoque de las políticas farmacéuticas

Las dimensiones disponibilidad y asequibilidad son determinantes para el acceso, pues permiten que el bien o servicio esté presente para su uso. La disponibilidad depende, en gran medida, de los procesos iniciales de la cadena terapéutica: Investigación y Desarrollo $(\mathrm{I}+\mathrm{D})$, fabricación, autorización, distribución y comercialización. La accesibilidad a un medicamento se encuentra relacionada con el proceso de dispensación, pues es fundamental que el paciente pueda dirigirse al servicio farmacéutico a solicitar sus medicamentos. La aceptabilidad favorece el uso racional de medicamentos durante los procesos de prescripción y administración/uso. Dentro de la cadena terapéutica también se tiene la evaluación de resultados terapéuticos, que permite mejorar el uso racional de medicamentos. La calidad, siendo transversal a todas las dimensiones y todos los enfoques, propende por el cumplimiento de las especificaciones de cada proceso, para garantizar la seguridad de los pacientes y los mejores resultados en salud, y puede establecerse mediante la definición y el seguimiento de indicadores.

En Colombia, se cuenta con la "Política Farmacéutica Nacional" (PFN) [9], desde la que el Ministerio de Salud y Protección Social (MSPS) ha trabajado para favorecer el acceso a medicamentos. Una muestra de esto es que, para el 2016, se estimaba que el PBs cubría el 61,3\% de los medicamentos incluidos en el listado de medicamentos esenciales número 19 de la oms [10]. Sin embargo, algunos fármacos han permanecido por fuera del PBS, especialmente por su alto costo, representando una barrera de acceso para los pacientes, quienes han optado por recurrir al uso de herramientas legales, como las tutelas y la evaluación por comités técnico-científicos, para ejercer su derecho

* Listados que definen de manera explícita las tecnologías en salud que serán cubiertas con recursos públicos, para evitar el racionamiento poco equitativo e ineficiente de los servicios sanitarios [3]. 
a la salud [11]. Por ejemplo, para el 2012, se habían reportado, en la literatura, entre 11,9 y $35,6 \%$ de demandas relacionadas con medicamentos [12].

El uso de medicamentos de alto costo - aquellos por fuera del PBs o que hacen parte de las enfermedades de alto costo definidas por el MSPS - ha supuesto un aumento en el gasto del presupuesto en salud, como lo mostró un estudio realizado en 20 ciudades del país, en el que se analizaron los datos de fórmulas de medicamentos de alto costo dispensadas entre los años 2005 y 2010, en donde hubo un crecimiento del $847 \%$ en el valor facturado por estos medicamentos durante el periodo estudiado [11]. Esto plantea la necesidad de continuar implementando estrategias que favorezcan el acceso a medicamentos, con el fin de disminuir la carga de la enfermedad en la población y la carga económica para el sistema de salud colombiano.

En este sentido, el objetivo de esta revisión es identificar estrategias que favorezcan el acceso a medicamentos de interés en salud pública, de alto costo o protegidos por patentes, que tengan posibilidad de aplicación en el contexto colombiano.

\section{Metodología}

\section{Criterios de eligibilidad}

Se tomaron en cuenta artículos originales, revisiones, comentarios, editoriales, cartas al editor, libros y documentos que presentaran estrategias o políticas que favorecieran el acceso a medicamentos de interés en salud pública, ya fueran de alto costo o protegidos por patentes. Se excluyeron artículos que no estuvieran relacionados con el objetivo de la búsqueda.

\section{Fuentes de información y búsqueda}

En mayo de 2017 se realizó la búsqueda de artículos publicados en PubMed/Medline, utilizando los términos: ("health services accessibility"[MeSH Terms] OR "access to medicines"[All Fields]) AND ("pharmaceutical preparations"[MeSH Terms] OR “drugs"[All Fields]) AND ("policy”[MeSH Terms] or "policy"[All Fields].

Se estableció un límite de fechas entre el $1 .^{\circ}$ de mayo de 2012 y el $1 .^{\circ}$ de mayo de 2017 , en idiomas inglés y español.

\section{Selección de artículos}

Inicialmente, se revisaron el título y el resumen de los artículos de forma pareada, se eliminaron los artículos duplicados y aquellos no relacionados con el objetivo de la búsqueda. Posteriormente, se hizo una lectura de los textos preseleccionados. La búsqueda se complementó con referencias adicionales que incluyen el "Informe del Panel de Alto Nivel para el Acceso a Medicamentos del secretario general de la Organización de la Naciones Unidas" [4], la "Política Farmacéutica de Colombia" [9], la "Declaración de Doha" [13] y publicaciones referenciadas en los artículos revisados.

\section{Recolección de la información}

De cada artículo elegido se extrajo información sobre los autores, tipo de publicación, año de publicación, estrategias mencionadas o aplicadas, y el país de análisis/ aplicación de las estrategias.

Las estrategias identificadas se agruparon y clasificaron dentro de las cinco dimensiones que definen el acceso a la atención en salud y medicamentos según la oms (véase tabla 1) [8]. Dichas categorías se utilizaron en las fases de recolección de la información y análisis.

Tabla 1. Dimensiones del acceso a la atención en salud, medicamentos, vacunas y otros servicios de salud.

\begin{tabular}{|c|c|}
\hline Dimensión & Definición \\
\hline Disponibilidad & $\begin{array}{l}\text { Relación entre el tipo/calidad del producto/servicio requerido y el tipo/calidad de producto/servicio } \\
\text { entregado }\end{array}$ \\
\hline Asequibilidad & Relación entre el precio del producto/servicio y la capacidad del usuario para pagar por él \\
\hline Accesibilidad & Relación entre la ubicación de un producto/servicio y la ubicación de un eventual usuario que los requiere \\
\hline $\begin{array}{l}\text { Aceptabilidad } \\
\text { (o satisfacción) }\end{array}$ & $\begin{array}{l}\text { Relación entre las actitudes y expectativas del usuario (prescriptor o paciente) y las características reales de } \\
\text { los productos o servicios }\end{array}$ \\
\hline Calidad & $\begin{array}{l}\text { Relacionada con el cumplimiento de las especificaciones establecidas por los entes reguladores en } \\
\text { términos de seguridad, eficacia y costo-efectividad. Es transversal a todas las dimensiones }\end{array}$ \\
\hline
\end{tabular}

Fuente: [8]. 


\section{Resultados}

\section{Selección de artículos}

Se identificaron 62 referencias, de los cuales se incluyeron 18 y también se juzgó importante incluir 5 publicaciones adicionales (véase figura 2).

\section{Características de los artículos}

El $56,5 \%$ de las publicaciones incluidas fueron revisiones, seguidas de editoriales $(17,4 \%)$, políticas $(13,0 \%)$, artículos originales $(8,7 \%)$ y reportes $(4,4$ $\%)$. El $65,2 \%$ de los artículos fueron publicados entre 2014 y 2016.

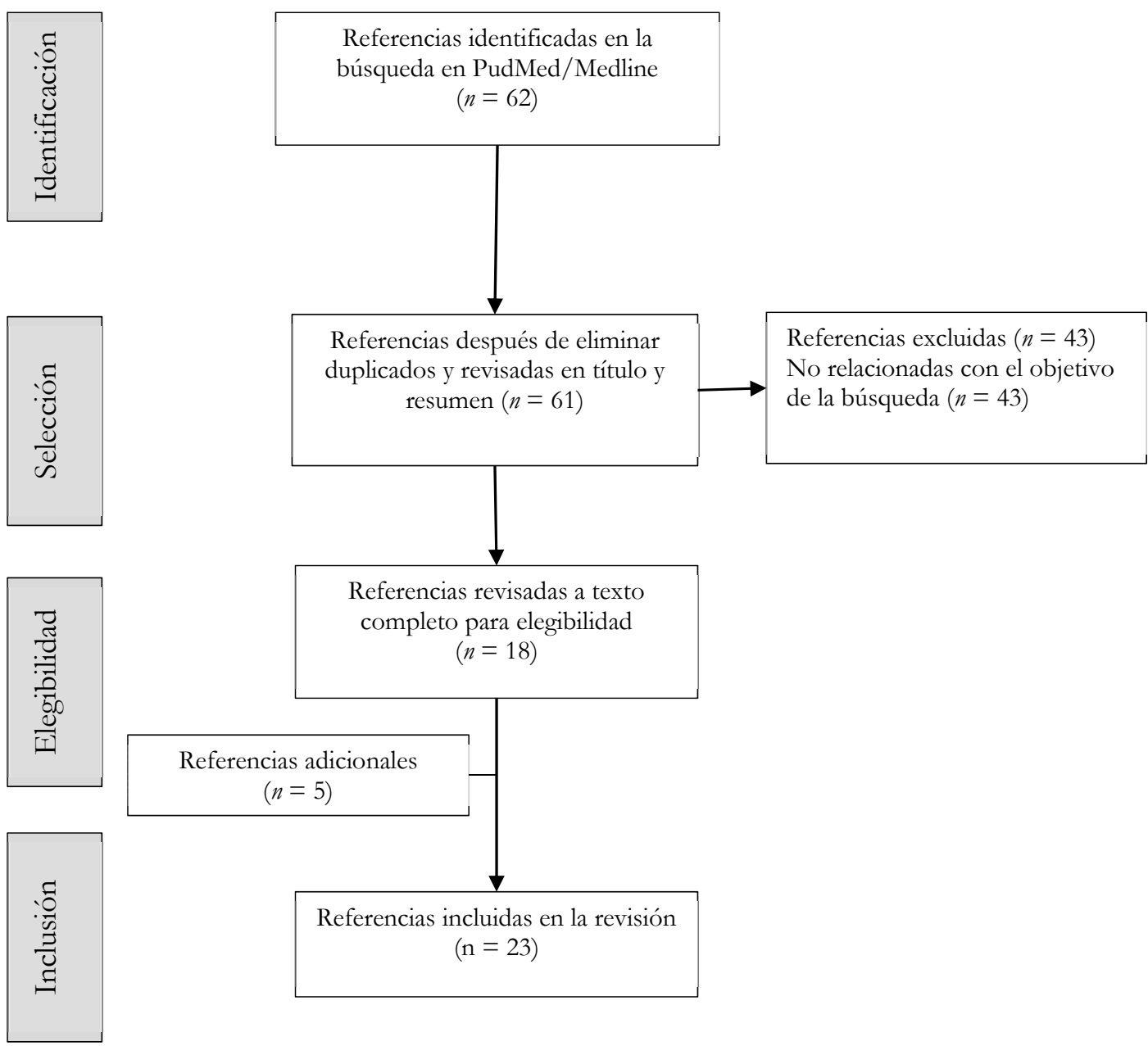

Figura 2. Diagrama de flujo de las referencias seleccionadas para la revisión

El 47,9 \% de los manuscritos abordaban las estrategias desde una perspectiva global, el 13,0\% se enfocó en estrategias a desarrollar en países de ingresos medios y bajos, el 8,7 \% se refirió a estrategias que han sido implementadas en Estados Unidos y el 8,7 \% eran políticas establecidas en Colombia. El 21,7 \% restante se dividió en artículos que estudiaron las estrategias de acceso a medicamentos en Brasil, Holanda, Escocia, India y Sudáfrica (véase tabla 2). 
Tabla 2. Referencias incluidas en la revisión.

\begin{tabular}{|c|c|c|c|c|c|}
\hline Título & $\begin{array}{c}\text { Tipo de } \\
\text { publicación }\end{array}$ & $\begin{array}{c}\text { Año de } \\
\text { publicación }\end{array}$ & Autor & $\begin{array}{c}\text { País de los } \\
\text { autores }\end{array}$ & $\begin{array}{c}\text { País de aplicación/ } \\
\text { análisis de las estrategias }\end{array}$ \\
\hline $\begin{array}{l}\text { Limiting the access to direct-acting } \\
\text { antivirals against HCV: An ethical } \\
\text { dilemma [14]. }\end{array}$ & Revisión & 2016 & $\begin{array}{l}\text { Gentile I, Maraolo } \\
\text { AE, Niola M, } \\
\text { Graziano V, Borgia } \\
\text { G, Paternoster M. }\end{array}$ & Italia & Global \\
\hline $\begin{array}{l}\text { The impact of the legal regime of } \\
\text { intellectual property protection in the } \\
\text { pharmaceutical market [15]. }\end{array}$ & Revisión & 2016 & $\begin{array}{l}\text { Pashkov VM, } \\
\text { Golovanova IA, } \\
\text { Olefir AA. }\end{array}$ & Ucrania & $\begin{array}{l}\text { Global, exponen el caso } \\
\text { de India }\end{array}$ \\
\hline $\begin{array}{l}\text { Access to medications for } \\
\text { cardiovascular diseases in low- and } \\
\text { middle-income countries [16]. }\end{array}$ & Revisión & 2016 & $\begin{array}{l}\text { Wirtz VJ, Kaplan WA, } \\
\text { Kwan GF, Laing RO. }\end{array}$ & EE.UU & $\begin{array}{l}\text { LMIC, exponen el caso de } \\
\text { México }\end{array}$ \\
\hline $\begin{array}{l}\text { Overcoming obstacles to } \\
\text { enable access to medicines for } \\
\text { noncommunicable diseases in poor } \\
\text { countries [17]. }\end{array}$ & Revisión & 2015 & $\begin{array}{l}\text { Kishore SP, Kolappa } \\
\text { K, Jarvis JD, } \\
\text { Park PH, Belt R, } \\
\text { Balasubramaniam } \\
\text { T, et al. }\end{array}$ & EE.UU & $\begin{array}{l}\text { Global, menciona casos } \\
\text { de algunos países como } \\
\text { India, EE. UU., Grecia, } \\
\text { España, Dinamarca }\end{array}$ \\
\hline $\begin{array}{l}\text { Access to Costly New Hepatitis } \\
\text { C Drugs: Medicine, Money, and } \\
\text { Advocacy [18]. }\end{array}$ & Editorial & 2015 & $\begin{array}{l}\text { Trooskin SB, } \\
\text { Reynolds H, } \\
\text { Kostman JR. }\end{array}$ & EE.UU. & EE. UU. \\
\hline $\begin{array}{l}\text { Expansion of hcv treatment access } \\
\text { to people who have injected drugs } \\
\text { through effective translation of } \\
\text { research into public health policy: } \\
\text { Scotland's experience [19]. }\end{array}$ & $\begin{array}{l}\text { Artículo } \\
\text { original }\end{array}$ & 2015 & $\begin{array}{l}\text { Hutchinson SJ, } \\
\text { Dillon JF, Fox R, } \\
\text { McDonald SA, Innes } \\
\text { HA, Weir A, et al. }\end{array}$ & Escocia & Escocia \\
\hline $\begin{array}{l}\text { Assessing } 15 \text { proposals for } \\
\text { promoting innovation and access to } \\
\text { medicines globally [20]. }\end{array}$ & Revisión & 2014 & $\begin{array}{l}\text { Hoffman SJ, So K; } \\
\text { Global Access to } \\
\text { Medicines Reform } \\
\text { Study Team. }\end{array}$ & Varios & Global \\
\hline $\begin{array}{l}\text { Societal value of generic medicines } \\
\text { beyond cost-saving through reduced } \\
\text { prices [21]. }\end{array}$ & Revisión & 2015 & $\begin{array}{l}\text { Dylst P, Vulto A, } \\
\text { Simoens S. }\end{array}$ & $\begin{array}{l}\text { Bélgica, } \\
\text { Holanda }\end{array}$ & Holanda \\
\hline $\begin{array}{l}\text { Production of antiretroviral drugs in } \\
\text { middle- and low-income countries } \\
\text { [22]. }\end{array}$ & Revisión & 2014 & $\begin{array}{l}\text { Pinheiro EdS, } \\
\text { Brüning K, Macedo } \\
\text { MF, Siani AC. }\end{array}$ & Brasil & $\begin{array}{l}\text { LMIC-MIC: Menciona } \\
\text { casos de Brasil, Argentina, } \\
\text { China e India. }\end{array}$ \\
\hline $\begin{array}{l}\text { Intellectual property rights, market } \\
\text { competition and access to } \\
\text { affordable antiretrovirals [23]. }\end{array}$ & Revisión & 2014 & Pascual F. & España & $\begin{array}{l}\text { Global; menciona caso } \\
\text { de Argentina e India y } \\
\text { algunos paises de África }\end{array}$ \\
\hline $\begin{array}{l}\text { Changes to intellectual property } \\
\text { policy in South Africa: Putting a stop } \\
\text { to evergreening? [24] }\end{array}$ & Editorial & 2015 & Hill JE. & Sudáfrica & Sudáfrica \\
\hline $\begin{array}{l}\text { Hepatitis C, a global issue: Access } \\
\text { to care and new therapeutic and } \\
\text { preventive approaches in resource- } \\
\text { constrained areas [25]. }\end{array}$ & Revisión & 2014 & $\begin{array}{l}\text { Lemoine } M \text {, Thursz } \\
\text { M. }\end{array}$ & Reino Unido & Global \\
\hline $\begin{array}{l}\text { Decentralising HIV treatment in } \\
\text { lower- and middle-income countries } \\
\text { [26]. }\end{array}$ & Revisión & 2013 & $\begin{array}{l}\text { Kredo T, Ford N, } \\
\text { Adeniyi FB, Garner P. }\end{array}$ & Sudáfrica & LMIC \\
\hline $\begin{array}{l}\text { Hepatitis } C \text { treatment access and } \\
\text { uptake for people who inject drugs: } \\
\text { A review mapping the role of social } \\
\text { factors [27]. }\end{array}$ & Revisión & 2013 & Harris M, Rhodes T. & Reino Unido & Global \\
\hline
\end{tabular}




\begin{tabular}{|c|c|c|c|c|c|}
\hline Título & $\begin{array}{c}\text { Tipo de } \\
\text { publicación }\end{array}$ & $\begin{array}{c}\text { Año de } \\
\text { publicación }\end{array}$ & Autor & $\begin{array}{l}\text { País de los } \\
\text { autores }\end{array}$ & $\begin{array}{c}\text { País de aplicación/ } \\
\text { análisis de las estrategias }\end{array}$ \\
\hline $\begin{array}{l}\text { Is India ready to lead the battle for } \\
\text { fair access to medicines? [28]. }\end{array}$ & Editorial & 2013 & $\begin{array}{l}\text { The Lancet } \\
\text { Oncology. }\end{array}$ & India & India \\
\hline $\begin{array}{l}\text { Promotion of access to essential } \\
\text { medicines for non-communicable } \\
\text { diseases: Practical implications of } \\
\text { the UN political declaration [29]. }\end{array}$ & Revisión & 2013 & $\begin{array}{l}\text { Hogerzeil HV, } \\
\text { Liberman J, Wirtz VJ, } \\
\text { Kishore SP, Selvaraj } \\
\text { S, Kiddell-Monroe } \\
\text { R, et al. }\end{array}$ & Holanda & Global \\
\hline $\begin{array}{l}\text { Proposed "grant-and-access" } \\
\text { program with price caps could } \\
\text { stimulate development of drugs for } \\
\text { very rare diseases [30]. }\end{array}$ & Revisión & 2012 & $\begin{array}{l}\text { Valverde AM, Reed } \\
\text { SD, Schulman KA. }\end{array}$ & EE.UU & EE.UU \\
\hline $\begin{array}{l}\text { Emergency contraception: Global } \\
\text { challenges, new opportunities [31]. }\end{array}$ & Editorial & 2012 & $\begin{array}{l}\text { Westley E, Schwarz } \\
\text { EB. }\end{array}$ & EE.UU & Global \\
\hline $\begin{array}{l}\text { Strategies for price reduction of } \\
\text { hiv medicines under a monopoly } \\
\text { situation in Brazil [32]. }\end{array}$ & $\begin{array}{l}\text { Artículo } \\
\text { original }\end{array}$ & 2015 & $\begin{array}{l}\text { Chaves GC, } \\
\text { Hasenclever L, } \\
\text { Osorio-de-Castro } \\
\text { CG, Oliveira MA. }\end{array}$ & Brasil & Brasil \\
\hline $\begin{array}{l}\text { Final Report. The United Nations } \\
\text { Secretary-General's High-Level Panel } \\
\text { on Access to Medicines [4]. }\end{array}$ & Reporte & 2016 & $\begin{array}{l}\text { Organización de las } \\
\text { Naciones Unidas }\end{array}$ & Varios & Global \\
\hline Política Farmacéutica Nacional [9]. & Política & 2012 & $\begin{array}{l}\text { Consejo Nacional de } \\
\text { Política Económica } \\
\text { y Social }\end{array}$ & Colombia & Colombia \\
\hline Plan Nacional de Desarrollo [33]. & Política & 2015 & $\begin{array}{l}\text { Congreso de la } \\
\text { República de } \\
\text { Colombia. }\end{array}$ & Colombia & Colombia \\
\hline Declaración de Doha [13]. & Política & 2011 & $\begin{array}{l}\text { Organización } \\
\text { Mundial de la Salud }\end{array}$ & Varios & Global \\
\hline
\end{tabular}

EE.UU.: Estados Unidos de América; LMIC: Low and Middle Income Countries (Países de bajos y medianos ingresos); MIC: Middle Income Countries (Países de medianos ingresos).

\section{Estrategias para promover el acceso a medicamentos de interés en salud pública}

Las estrategias o las políticas encontradas en las publicaciones revisadas se resumieron en treinta estrategias, las cuales se agruparon bajo las cinco dimensiones del acceso, teniendo en consideración la transversalidad de ellas en varias dimensiones.

\section{Estrategias para mejorar la disponibilidad y la asequibilidad}

- Crear apoyos financieros e incentivos públicos (premios [17,20,24], subsidios, compromisos de compra anticipada [20], reducción de impuestos o precios de los servicios públicos [15]) para la I+D nacional, con el fin de incrementar la disponibilidad interna de ingredientes farmacéuticos activos $[15,22,32]$ y de medicamentos genéricos de bajo precio y con calidad asegurada, que respondan a las necesidades de salud insatisfechas $[4,9,21,24,29]$ y fortalezcan el potencial de exportación $[15,22]$. El conocimiento generado por esta $\mathrm{I}+\mathrm{D}$ debe ser de libre acceso, con el fin de intercambiar conocimientos y tecnologías que faciliten el desarrollo de nuevos productos en salud $[4,17,20]$. En contrapartida, las compañías farmacéuticas podrían desvincular el precio final de las tecnologías en salud del costo de la I+D $[4,17,20,24]$; en este sentido, el precio podría establecerse basado en la duración de los tratamientos, el tamaño esperado del mercado y las tasas de retorno [30].

- Buscar el apoyo de organismos internacionales (Organización Mundial de la salud, Organización de las Naciones Unidas) para la financiación de proyectos de I+D nacional, que permitan la producción de medicamentos de interés en salud pública que más necesita el país [29]. Adicionalmente, estas organizaciones podrían salvaguardarlo de 
la influencia de las multinacionales en temas relacionados con el acceso a medicamentos [17].

- Establecer acuerdos multilaterales en salud con los países vecinos, que incluyan transferencias de tecnologías, como los ingredientes farmacéuticos activos [22].

- Aplicar las flexibilidades del acuerdo sobre los "Aspectos de los derechos de propiedad intelectual relacionados con el comercio" (ADPIC), reafirmadas en la "Declaración de Doha": licencias obligatorias, excepción bolar* e importaciones paralelas [4,13$15,17,23,25,28,29,32]$.

- Modificar las normativas que regulan el otorgamiento de patentes, aplicando estándares más rigurosos de invención, que estén a favor de los intereses en salud pública $[4,15,23,24,28,29]$ y eviten el reverdecimiento de patentes [4,24]. Podría restringirse la protección patentada de ME o huérfanos $[15,16]$.

- Negociar licencias voluntarias con los titulares de las patentes, mediante la participación en fondos comunes de patentes (Medicines Patent Pool MPP-) $[4,17,20,23,25,32]$.

- Utilizar las patentes sin expedir licencia voluntaria para la fabricación de medicamentos genéricos y pagar regalías al titular de la patente $[18,23]$.

- Realizar negociaciones centralizadas de precios $[9,14,18,32,33]$.

- Adoptar sistemas de referenciación internacional de precios $[9,14,16,23,32]$.

- Crear un organismo central que coordine una adquisición global y coordinada de medicamentos a granel [17].

- Aplicar exención de impuestos aduaneros y de ventas a los medicamentos para enfermedades de interés en salud pública que sean importados $[16,17]$.

- Eliminar los datos de exclusividad, para favorecer la aprobación de medicamentos genéricos y que así estos puedan estar disponibles a precios más bajos inmediatamente después de la expiración de las patentes [20].

- Revisar conjuntamente, entre los ministerios de Comercio y Salud, los acuerdos internacionales para evaluar el impacto en la salud pública [4].

- Incrementar la financiación de medicamentos [16], por medio de impuestos o esquemas de seguros [29].

- Asegurar procesos transparentes y responsables, en los que se identifiquen explícitamente los conflictos de intereses y se evite su influencia en la instauración de políticas y adquisición de medicamentos [29].

* Excepción legal que permite el uso de una patente de invención antes de su expiración, con el fin de obtener la autorización necesaria para comercializar el producto genérico cuando expire la patente correspondiente [4].

\section{Estrategias para mejorar la accesibilidad}

- Mejorar la logística y los canales de distribución de los medicamentos [9], especialmente en las zonas rurales.

- Disminuir los tiempos de espera de entrega de medicamentos, agilizando los procesos administrativos y generando cambios en las regulaciones [16].

- Contar con flexibilidad de horarios para la entrega de medicamentos [27].

- Aumentar la calidad de la atención y generar indicadores para monitorear los cambios e identificar las necesidades de los pacientes [16].

- Descentralizar la atención en salud y ampliar el suministro de medicamentos a centros de salud periféricos o incluso más allá de los centros de salud [26].

- Ofrecer asistencia cuando haya dificultades en transporte, alojamiento y acceso a beneficios sociales [27].

\section{Estrategias para mejorar la aceptabilidad}

- Establecer una atención primaria centrada en el paciente, con el fin de mejorar su adherencia al tratamiento [27-29].

- Crear una entidad nacional, responsable de la promoción del uso eficiente y seguro de los medicamentos, además del monitoreo de la adherencia de los pacientes [29]. Para promover y monitorear la adherencia, pueden emplearse terapias de observación directa y sistemas electrónicos de recordatorio para la toma de medicamentos [27].

- Proveer combinaciones de dosis fijas de medicamentos, para favorecer la adherencia a los tratamientos $[16,17]$.

- Usar redes sociales, mensajes de texto, internet, radio, televisión y otros medios para aumentar la conciencia sobre el acceso a medicamentos y la importancia de su uso adecuado [31].

- Capacitar al personal de salud de atención primaria en el manejo adecuado de los medicamentos y el acceso a los mismos [27].

- Apoyar financieramente a la sociedad civil, para promover la defensa del acceso de medicamentos. Dicha financiación puede provenir de los impuestos al tabaco o las aerolíneas, en lugar de la industria farmacéutica, reduciendo así la dependencia a fuentes como los laboratorios farmacéuticos y, a su vez, el conflicto de intereses [4,17,29].

\section{Estrategias para mejorar la calidad}

- Utilizar las nuevas tecnologías de comunicación para monitorear las reservas de medicamentos en zonas rurales o remotas [29]. 
- Seguir buenas prácticas de adquisición y verificar la identidad de los productos [16].

\section{Estrategia común a todas las dimensiones}

- Desarrollar programas de asistencia a pacientes vulnerables [19], con enfermedades de interés en salud pública o medicamentos de alto costo $[9,18]$. Estos programas deben tener un enfoque multidisciplinario, especialmente cuando se trata a pacientes vulnerables, como usuarios de drogas inyectables [27], prisioneros, entre otros.

\section{Experiencias de aplicación de algunas de las estrategias identificadas.}

\section{Producción local de medicamentos genéricos}

En materia de ARV, varios países cuentan con la capacidad para producir medicamentos genéricos para satisfacer sus demandas internas de estos ME. Tal es el caso de Argentina, Brasil y Cuba en América Latina, e India, China y Tailandia en Asia. Algunos países de África, como Ghana, Kenia, Nigeria, República Democrática del Congo, Sudáfrica, Tanzania, Uganda, Zambia y Zimbabue, cuentan con industrias manufactureras, algunas de ellas asociadas a farmacéuticas alemanas, chinas o indias, para la producción local de ARV [22].

Utilización de las flexibilidades del acuerdo sobre los ADPIC

India ha sido un gran ejemplo de utilización de las flexibilidades del acuerdo sobre los ADPIC, en especial en la concesión de licencias obligatorias para permitir el acceso a los medicamentos de interés en salud pública, otorgadas principalmente durante la crisis de salud. Uno de los casos se dio en marzo de 2012, cuando el contralor general de Patentes, Diseños y Marcas emitió una licencia obligatoria sobre sorafenib, un medicamento para tratar el carcinoma renal y hepatocelular $[17,28,29]$, que permitió la producción y la comercialización de su versión genérica antes de que expirara la patente, a un precio mucho más bajo que el que presentaba en los países occidentales (150 vs. 4000 euros) [25]. Entre los motivos citados por las autoridades indias al otorgar la licencia obligatoria a Natco Pharma, un fabricante indio de genéricos, estaba el hecho de que el sorafenib no estaba "disponible para el público a un precio razonablemente asequible" [17,29] y que la compañía farmacéutica Bayer no había garantizado la disponibilidad de cantidades suficientes en India [29].

Así mismo, países de medianos y bajos ingresos, como Brasil, Ecuador, Indonesia, Malasia, Mozambique y Tailandia, han otorgado licencias obligatorias o han hecho uso de medicamentos ARV patentados (bajo uso gubernamental), después de que el acuerdo sobre los
ADPIC tomó fuerza. Por ejemplo, en septiembre de 2012, Indonesia otorgó una licencia obligatoria, autorizando la producción local, importación y venta de versiones genéricas de siete medicamentos patentados usados en el VIH y en el virus de la hepatitis B, con un pago de regalías del $0,5 \%$ del valor neto de venta de los medicamentos bajo licencia [23].

\section{Regulación del otorgamiento de patentes}

India y Argentina han adoptado criterios más rigurosos para el otorgamiento de patentes, demostrando que existe un rango de maniobra al que pueden acceder los países que están bajo el acuerdo sobre los ADPIC. Dentro de dichos criterios puede estar la definición de qué es patentable y qué puede ser patentado [23].

En India, se han redefinido los estándares de patentabilidad en la sección 3 de la Ley de Patentes de 2005, que incluye una cláusula para evitar el reverdecimiento de patentes, al excluir la patentabilidad de sustancias conocidas modificadas, a menos que la modificación dé como resultado una eficacia mejorada [24]; esto se hizo con el fin de salvaguardar el interés público y mantener un equilibrio para el acceso de los medicamentos que salvan vidas y que se calificaban como bienes públicos [17]. Bajo dicha premisa, India negó la patente de la sal cristalina de imatinib (mesilato de imatinib, comercializado como Glivec ${ }^{\circledR}$ ) a Novartis, dado que la oficina de Patentes dictaminó que Glivec ${ }^{\circledR}$ no mostró ninguna ventaja significativa en comparación con la forma anterior del imatinib [29].

Algunos países incluyen, en sus leyes de patentes, el permitir que terceras partes disputen la validez de una solicitud de patente o de una patente ya otorgada, cuando se estima un incumplimiento de los criterios de patentabilidad. Por ejemplo, en India, a raíz de múltiples oposiciones a solicitudes de patentes, se rechazó el otorgamiento de la patente al tenofovir disoproxil fumarato, un ARV clave en los regímenes de primera línea para el VIH. Como resultado, las industrias de genéricos en la India pueden producir y exportar este medicamento en países donde no está patentado [23].

Argentina también ha adoptado nuevas directrices de evaluación de patentes, que contienen criterios de exclusión más exhaustivos que en la India, y no incluyen disposiciones de "eficacia mejorada". Bajo esta interpretación, si un compuesto farmacéutico es parte del estado de la técnica, las consideraciones de salud pública para el acceso a medicamentos genéricos más asequibles superan los intereses comerciales de la compañía creadora. En los 5 meses anteriores a la entrada en vigor de las directrices, Argentina otorgó 53 patentes farmacéuticas, mientras que en los 7 meses siguientes otorgó solo dos [24]. 
Adquisición coordinada de medicamentos por una organización central

La experiencia del VIH/SIDA ha demostrado que la adquisición global y coordinada de medicamentos a granel por parte de una organización central es una forma efectiva de bajar los precios y mejorar la disponibilidad para un gran número de personas. Una muestra de ello es el 'US President's Emergency Plan for AIDS Relief" que, desde el 2003, lleva a cabo adquisiciones conjuntas para medicamentos contra el VIH/SIDA, y la "Stop TB Partnership" que, desde el 2001, ayuda a realizar compras conjuntas de medicamentos clave para la tuberculosis en Estados Unidos. El "Asthma Drug Facility", administrado por la Unión Internacional contra la Tuberculosis y la Enfermedad Pulmonar, y que inició en 1998, hace lo mismo con los inhaladores para el asma. La Organización Panamericana de la Salud, un brazo regional de la oms, tiene un fondo que proporciona medicamentos a granel a los países que lo integran en la región panamericana [17].

\section{Programas de asistencia}

En Estados Unidos se han implementado programas de asistencia de medicamentos para abordar la epidemia de VIH/SIDA, que han contribuido al mejoramiento de los resultados de salud y reducido drásticamente los costos de tratamiento. Debido a su éxito, se ha propuesto la creación de un programa para el tratamiento del VHC, que permitiría la negociación de precios, la contención de costos y el aumento del acceso a AAD. Inclusive, manifiestan que estos programas podrían combinarse con una adquisición de la patente voluntaria de Gilead (fabricante de algunos AAD) por parte del Gobierno de Estados Unidos [18].

Otro programa instaurado por un gobierno es el "Hepatitis C Action Plan", en Escocia, que ha logrado un aumento cercano al $50 \%$ en el diagnóstico de la población infectada con VHC y un incremento en más del doble de las personas que inician tratamiento, especialmente en usuarios de drogas inyectables y prisioneros [19]. Así mismo, en Brasil se ha implementado un programa para el manejo del VIH, que desde 1996 garantiza el acceso universal gratuito a los medicamentos ARV. Para lograrlo, el Gobierno brasilero ha recurrido a la negociación de precios con empresas farmacéuticas multinacionales, con la amenaza de emitir licencias obligatorias. También ha establecido una referenciación internacional de precios, expedición de licencias obligatorias para la importación y la posterior producción local del medicamento, participación en MPP, entre otras, logrando reducciones hasta del $50 \%$ en el precio de medicamentos, como atazanavir [32].

\section{Discusión}

La inequidad y el acceso limitado a los medicamentos son un indicador de las fallas en los sistemas de salud y en las políticas farmacéuticas [34], que hacen necesario el establecimiento de estrategias a corto y largo plazo que contribuyan al acceso equitativo a medicamentos efectivos, a la prestación de servicios de atención en salud de calidad y, por tanto, al mejoramiento de las condiciones de salud de la población.

En la presente revisión se identificaron treinta estrategias claves, aplicables para mejorar el acceso a medicamentos de interés en salud pública de alto costo o protegidos por derechos de propiedad intelectual, desde las cinco dimensiones del acceso: disponibilidad, asequibilidad, accesibilidad, aceptabilidad y calidad. Estas estrategias pueden ser aplicadas en el actual contexto colombiano, siendo útiles para el Gobierno y las instituciones encargadas de la formulación de políticas públicas en materia de salud y propiedad intelectual, así como para las empresas aseguradoras y prestadoras de servicios de atención en salud. Su implementación requiere la articulación del Gobierno con las instituciones públicas y privadas, en aras de priorizar el bienestar de la comunidad sobre los intereses comerciales particulares y la reducción del gasto farmacéutico.

La instauración de las estrategias identificadas representaría grandes beneficios para la población, las empresas prestadoras de servicios y el sistema de salud. La reducción en el precio de los medicamentos y la instauración de procesos encaminados a mejorar la aceptabilidad y la accesibilidad favorecerían el alcance de los objetivos terapéuticos y la mejora en la calidad de vida de los pacientes, al garantizar el acceso y la continuidad de los tratamientos. De igual forma, reduciría los gastos de bolsillo en los que los pacientes incurren por concepto de copagos y trámites para acceder a medicamentos de alto costo. Para las empresas prestadoras de servicios, la aplicación de las estrategias relacionadas con la accesibilidad y la aceptabilidad permitiría optimizar los procesos, reduciendo costos y ampliando la cobertura de atención. En el caso del sistema de salud, se generaría un ahorro en el gasto farmacéutico nacional, pudiendo destinar los recursos a la atención de otras necesidades básicas de la población.

La mayoría de los autores mencionaron estrategias dirigidas a mejorar la disponibilidad y la asequibilidad de los medicamentos, ratificando la importancia de fortalecer la $I^{+} D$ nacional, a través de apoyos financieros e incentivos públicos que estimulen la producción de principios activos y medicamentos genéricos. Esto depende por completo de las políticas instauradas por los gobiernos, y requiere el desarrollo de programas que incentiven la investigación y la integración entre la 
academia y la industria, para que los descubrimientos realizados en centros de investigación universitarios se materialicen a gran escala en la industria farmacéutica nacional. Como lo menciona Dylst et al., los beneficios de la fabricación nacional de medicamentos genéricos para enfermedades de alto costo trascienden la disminución de precios, pues también favorecen el acceso a la farmacoterapia y mejoran la relación costoefectividad de los tratamientos existentes. Esto mejora la rentabilidad en el manejo de pacientes no tratados previamente y amplía la cobertura para tratar otras patologías, utilizando el mismo presupuesto destinado a salud [21]

Otras intervenciones que buscan mejorar el acceso a medicamentos de interés en salud pública de alto costo son las negociaciones centralizadas de precios y la referenciación internacional. Ambas han sido incluidas dentro de la PFN y han sido utilizadas en Colombia. La primera se empleó en el proceso de compra centralizada de AAD para el manejo de la hepatitis C, a través del Fondo Rotatorio de la Organización Panamericana de la Salud [35], en la que se adquirieron inicialmente 1225 tratamientos y se logró la reducción en el precio de los medicamentos en un $80 \%$ [36]. La referenciación internacional comenzó a utilizarse en julio de 2013, al aplicar un régimen de control directo de precios para algunos medicamentos, teniendo como países de referencia a Alemania, Argentina, Australia, Brasil, Canadá, Chile, Ecuador, España, Estados Unidos, Francia, México, Noruega, Panamá, Perú, Portugal, Reino Unido y Uruguay [37]. Estas estrategias pueden ser utilizadas para más enfermedades de interés en salud pública, cuyos medicamentos sean de alto costo y dependerá de las negociaciones realizadas por el MSPS.

En el caso de los medicamentos protegidos por patentes, varios autores resaltan la importancia de aplicar las flexibilidades del acuerdo sobre los ADPIC, modificar las normativas que regulan el otorgamiento de patentes o participar en iniciativas que promueven el otorgamiento de licencias voluntarias como el MPP. En este sentido y considerando lo establecido en el artículo 31 del acuerdo sobre los ADPIC y en el capítulo VII de la "Decisión Andina 486 de 2000", Colombia, como miembro de la Organización Mundial del Comercio y de la Comunidad Andina, podría hacer uso de la patente de un medicamento cuando existan "razones de interés público", como los casos de emergencia nacional, o cuando se pretenda hacer un uso público no comercial (o uso por el Gobierno) del medicamento [38,39]. Esta herramienta no ha sido muy aplicada en el país y solo existe un antecedente de la aplicación de las flexibilidades del acuerdo sobre los ADPIC, específicamente para el caso del antineoplásico Glivec ${ }^{\circledR}$ (imatinib), el cual se declaró “de interés público" en la Resolución 2475 del 2016 [40] $\mathrm{y}$, por tanto, fue sometido al régimen de control directo de precios por parte de la Comisión Nacional de Precios de Medicamentos y Dispositivos Médicos.

Se encontraron pocos autores que abordaran a profundidad estrategias para mejorar la accesibilidad y la aceptabilidad de los medicamentos de interés en salud pública. Algunas de las recomendaciones a destacar y que podrían ser adoptadas por las empresas aseguradoras y prestadoras de servicios en Colombia son mejorar la logística y los canales de distribución de los medicamentos, además de descentralizar la atención en salud y ampliar el suministro, especialmente en zonas que se encuentran alejadas de los cascos urbanos. En este sentido, el MSPS, en el marco de la "Política de Atención Integral en Salud", ha incentivado el desarrollo de proyectos que buscan ampliar la atención en salud en zonas rurales o dispersas del territorio colombiano, por medio de servicios como la telemedicina y la telefarmacia [41,42]. Sin embargo, faltan mayores avances e inversión de recursos para garantizar la atención en salud y el acceso a medicamentos en dichas zonas.

Para fortalecer la dimensión de calidad, una de las recomendaciones es emplear las tecnologías de comunicación para monitorear las reservas de medicamentos en zonas rurales o dispersas. Aunque esta estrategia se contempló dentro de la PFN, se han presentado dificultades para su implementación. Actualmente, el MSPS está evaluando una propuesta de modelo de telefarmacia que podría mejorar la reserva de medicamentos y el acceso en estas zonas.

El desarrollo de programas nacionales de asistencia se identificó como una medida transversal a todas las dimensiones, dado que supone implementar varias de las estrategias identificadas en esta revisión, con el fin de resolver la problemática del acceso a medicamentos de interés en salud pública desde las cinco dimensiones. Esto requiere el esfuerzo mancomunado del Gobierno, la industria farmacéutica nacional, las empresas aseguradoras y los prestadores de servicios de salud.

En la creación de un programa nacional de asistencia podrían definirse, desde el Gobierno-MSPs, incentivos para la $\mathrm{I}+\mathrm{D}$ y fabricación de medicamentos genéricos en las industrias nacionales, y así suplir las necesidades de tratamientos a precios más asequibles. Además, las empresas aseguradores y prestadores participarían mediante la elaboración de: 1) protocolos para la promoción del tamizaje y detección temprana, con el consecuente inicio oportuno de los tratamientos; 2) campañas para prevenir la transmisión o el desarrollo de la enfermedad; 3) estrategias para aumentar la cobertura al acceso en zonas urbanas, rurales y dispersas; 4) acciones para incentivar el uso adecuado de medicamentos por parte de pacientes, prescriptores y personal de la salud en general, y 5) procesos para monitorear la seguridad y la efectividad de los medicamentos. 
Este es un proceso complejo que requiere la articulación de todos los actores del Sistema General de Seguridad Social en Salud y de la industria farmacéutica, pero que, a largo plazo, podría ayudar a resolver las dificultades económicas, administrativas, logísticas y asistenciales, que día a día enfrentan los pacientes en Colombia durante el proceso de atención. Los esfuerzos dirigidos a la gestión de la salud pública, la promoción de la salud y el diagnóstico, tratamiento y rehabilitación de las enfermedades de interés en salud pública permitirían reducir las tasas de transmisión de enfermedades como el VIH, el VHC o virus de la hepatitis B, y la aparición y la progresión de patologías crónicas. Así mismo, ampliaría la cobertura de acceso a tratamientos de alto costo y disminuiría la morbimortalidad asociada a las dificultades de acceso.

\section{Conclusiones}

La presente revisión estructurada resume estrategias para favorecer el acceso a medicamentos de interés en salud pública de alto costo o protegidos por DPI, utilizando las cinco dimensiones del acceso definidas por la oms: disponibilidad, asequibilidad, accesibilidad, aceptabilidad y calidad. De esta manera, dicha revisión ofrece un enfoque integral, orientado a la resolución de las necesidades en materia de medicamentos de interés en salud pública, que puede ser abordada por los entes gubernamentales encargados de proponer e instaurar políticas, hasta por los encargados de implementarlas, como son las empresas aseguradoras y los prestadores de servicios de salud.

Dentro de las estrategias que pueden ser aplicadas en Colombia están la ampliación de las negociaciones centralizadas de precios a otras enfermedades de alto costo y el apoyo financiero público para la investigación, el desarrollo y la producción nacional de medicamentos genéricos. En el caso de los medicamentos protegidos por DPI, se sugiere la aplicación de las flexibilidades del acuerdo sobre los ADPIC; la modificación de las normativas que regulan el otorgamiento de patentes a medicamentos desde la Superintendencia de Industria y Comercio, y la participación en iniciativas que promuevan el otorgamiento de licencias voluntarias, como el MPP.

De igual forma, se propone que desde el MSPs se instauren programas de asistencia en los que se involucre a todos los actores (industria, empresas aseguradoras y prestadoras de servicios, agrupaciones de pacientes), para que, desde su quehacer, desarrollen estrategias orientadas a mejorar las cinco dimensiones del acceso a medicamentos de interés en salud pública de alto costo o protegidos por patentes.

\section{Limitaciones}

Se presentan algunas limitaciones en la realización de este trabajo, dado que la búsqueda se llevó a cabo únicamente en la base de datos PubMed/Medline y lo recomendado es hacerlo en dos o más bases de datos. Sin embargo, esto pudo ser atenuado con la inclusión de revisiones que constituyen un $56 \%$ de las fuentes consultadas.

Adicionalmente, se tuvieron en cuenta referencias claves, como las recomendaciones del "Informe del Panel de Alto Nivel para el Acceso a Medicamentos del secretario general de la Organización de la Naciones Unidas" [4].

\section{Agradecimientos}

Al grupo de investigación Promoción y Prevención Farmacéutica de la Universidad de Antioquia, Medellín, Colombia

\section{Conflicto de intereses}

Los autores declaran no tener ningún conflicto de interés.

\section{Financiación}

El grupo Promoción y Prevención Farmacéutica recibió financiación de la Convocatoria de sostenibilidad 20182019, del Comité para el Desarrollo de la Investigación, de la Universidad de Antioquia.

\section{Declaración de responsabilidad}

Se declara que los puntos de vista expresados son responsabilidad de los autores y no de la institución en la que trabajan o de la fuente de financiación.

\section{Declaración de autoría}

Ledezma-Morales M: diseño de la revisión; revisión de artículos y extracción de información; análisis de la información; redacción del manuscrito inicial; revisión y aprobación de la versión final del manuscrito.

Amariles P: diseño de la revisión; revisión de artículos; análisis de la información; redacción del manuscrito inicial; revisión y aprobación de la versión final del manuscrito.

Vargas-Peláez CM: análisis de la información; redacción del manuscrito inicial; revisión y aprobación de la versión final del manuscrito.

Rossi Buenaventura FA: análisis de la información; redacción del manuscrito inicial; revisión y aprobación de la versión final del manuscrito. 


\section{Referencias}

1. World Health Organization. WHA28.66 Sustancias profilácticas y terapéuticas. 28. ${ }^{\text {a }}$ Asamblea Mundial de la Salud [internet]; 1975 [citado 2017 jun. 20]. Disponible en: http://apps.who.int/ medicinedocs/documents/s21447es/s21447es.pdf.

2. Bigdeli M, Jacobs B, Tomson G, et al. Access to medicines from a health system perspective. Health Policy Plan. 2013;28(7):692704. DoI: https://doi.org/10.1093/heapol/czs108.

3. Giedion U, Bitrán RA, Tristao I, editores. Planes de beneficios en salud de América Latina. Una comparación regional Washington: Inter-American Development Bank, Social Protection and Health Division; 2014.

4. United Nations. Final Report. The United Nations SecretaryGeneral's High-Level Panel on Access to Medicines [internet]. 2016 [citado 2017 may. 6]. Disponible en: http://www. unsgaccessmeds.org/final-report/.

5. World Health Organization. Monitoring Health for the SDGS Sustainable Development Goals [internet]. Geneva: World Health Organization [internet]; 2017 [citado 2018 ene. 30]. (World Health Statistics). Disponible en: http://apps.who.int/iris/ bitstream/10665/255336/1/9789241565486-eng.pdf.

6. World Health Organization. The World Medicines Situation Report [internet]. Geneva: World Health Organization; 2011 [citado 2018 ene. 30]. Disponible en: http://www.who.int/ medicines/areas/policy/world_medicines_situation/en/.

7. Gómez SM, Olaya P, Díaz FJ. Resistencia a los medicamentos antirretrovirales en pacientes que reciben tratamiento para $\mathrm{VIH}^{-}$ sida en Colombia. Infectio. 2010;14(4):248-57. DoI: https://doi. org/10.1016/S0123-9392(10)70118-1.

8. Management Sciences for Health. MDs-3: Managing Access to Medicines and Health Technologies [internet]. Arlington, va: Management Sciences for Healt [internet]; 2012 [citado 2017 jun. 20]. Disponible en: http://apps.who.int/medicinedocs/ documents/s19577en/s19577en.pdf.

9. Colombia, Consejo Nacional de Política Económica y Social. Política Farmacéutica Nacional. (2012 ago. 30). Disponible en: https://www. minsalud.gov.co/Documentos\%20y\%20Publicaciones/Politica\%20 Farmac\%C3\%A9utica\%20Nacional.pdf.

10. Colombia, Ministerio de Salud y Protección Social, Dirección de Regulación de Beneficios, Costos y Tarifas del Aseguramiento en Salud. Comparación entre el listado de medicamentos esenciales de la oms número 19 y el listado de medicamentos cubiertos por el Plan de Beneficios en Salud con cargo a la Unidad de Pago por Capitación definido mediante Resolución 5592 de 2015 [internet]; 2016. Disponible en: https://www.minsalud.gov.co/sites/rid/ Lists/BibliotecaDigital/RIDE/VP/RBC/Informe-medicamentosesenciales-oms-listado-medicamentos-pbsupc.pdf.

11. Machado Alba JE, Moncada Escobar JC. Evolución del consumo de medicamentos de alto costo en Colombia. Rev Panam Salud Pública. 2012;31(4):283-9. DoI: https://doi.org/10.1590/S102049892012000400003

12. Reveiz L, Chapman E, Torres R, et al. Litigios por derecho a la salud en tres países de América Latina: revisión sistemática de la literatura. Rev Panam Salud Pública. 2013;33(3):213-22. DoI: https://doi.org/10.1590/S1020-49892013000300008.

13. World Health Organization. Declaración de Doha [internet] 2001 [citado 2017 feb. 13]. Disponible en: www.wto.org/ spanish/res_s/booksp_s/ddec_s.pdf.

14. Gentile I, Maraolo AE, Niola M, et al. Limiting the access to direct-acting antivirals against HCV: An ethical dilemma. Expert
Rev Gastroenterol Hepatol. 2016;10(11):1227-34. DoI: https:// doi.org/10.1080/17474124.2016.1234375.

15. Pashkov VM, Golovanova IA, Olefir AA. The impact of the legal regime of intellectual property protection in the pharmaceutical market. Wiad Lek. 2016;69(3):582-6. Disponible en: https:// www.ncbi.nlm.nih.gov/pubmed/?term=27717949.

16. Wirtz VJ, Kaplan WA, Kwan GF, et al. Access to medications for cardiovascular diseases in low- and middle-income countries. Circulation. 2016;133(21):2076-85. DoI: https://doi.org/10.1161/ CIRCULATIONAHA.115.008722.

17. Kishore SP, Kolappa K, Jarvis JD, et al. Overcoming obstacles to enable access to medicines for noncommunicable diseases in poor countries. Health Aff (Millwood). 2015;34(9):1569-77. DoI: https://doi.org/10.1377/hlthaff.2015.0375.

18. Trooskin SB, Reynolds H, Kostman JR. Access to costly new hepatitis C drugs: Medicine, money, and advocacy. Clin Infect Dis. 2015;61(12):1825-30. DoI: https://doi.org/10.1093/cid/ civ677.

19. Hutchinson SJ, Dillon JF, Fox R, et al. Expansion of $\mathrm{HCV}$ treatment access to people who have injected drugs through effective translation of research into public health policy: Scotland's experience. Int J Drug Policy. 2015;26(11):1041-9. DOI: https://doi.org/10.1016/j.drugpo.2015.05.019.

20. Hoffman SJ, So K, Galappati P, et al. Assessing 15 proposals for promoting innovation and access to medicines globally. Ann Glob Health. 2014;80(6):432-43. DoI: http://doi.org/10.1016/j. aogh.2015.02.004.

21. Dylst P, Vulto A, Simoens S. Societal value of generic medicines beyond cost-saving through reduced prices. Expert Rev Pharmacoecon Outcomes Res. 2015;15(4):701-11. DoI: https:// doi.org/10.1586/14737167.2015.1017565

22. Pinheiro E dos S, Brüning K, Macedo MF, et al. Production of antiretroviral drugs in middle- and low-income countries. Antivir Ther. 2014;19(Suppl 3):49-55. DoI: https://doi.org/10.3851/ IMP2900.

23. Pascual F. Intellectual property rights, market competition and access to affordable antiretrovirals. Antivir Ther. 2014;19(Suppl 3):57-67. DoI: https://doi.org/10.3851/IMP2901.

24. Hill JE. Changes to intellectual property policy in South Africa: Putting a stop to evergreening? Expert Opin Ther Pat. 2014;24(8):839-43. DOI: https://doi.org/10.1517/13543776.201 4.931376 .

25. Lemoine M, Thursz M. Hepatitis C, a global issue: Access to care and new therapeutic and preventive approaches in resourceconstrained areas. Semin Liver Dis. 2014;34(01):089-97. DoI: https://doi.org/10.1055/s-0034-1371082.

26. Kredo T, Ford N, Adeniyi FB, et al. Decentralising HIV treatment in lower- and middle-income countries. En: The Cochrane Collaboration, editor. Cochrane Database of Systematic Reviews [internet]. Chichester, UK: John Wiley \& Sons, Ltd; 2013 [citado 2017 may. 18]. Disponible en: http://doi.wiley. com/10.1002/14651858.CD009987.pub2.

27. Harris $M$, Rhodes T. Hepatitis $C$ treatment access and uptake for people who inject drugs: A review mapping the role of social factors. Harm Reduct J. 2013;10:7. DoI: https://doi. org/10.1186/1477-7517-10-7.

28. The Lancet Oncology. Is India ready to lead the battle for fair access to medicines? Lancet Oncol. 2013;14(6):437. DoI: https:// doi.org/10.1016/S1470-2045(13)70197-9.

29. Hogerzeil HV, Liberman J, Wirtz VJ, et al. Promotion of access to essential medicines for non-communicable diseases: Practical 
implications of the UN political declaration. The Lancet 2013;381(9867):680-9. DoI: https://doi.org/10.1016/S01406736(12)62128-X.

30. Valverde AM, Reed SD, Schulman KA. Proposed "grantand-access" program with price caps could stimulate development of drugs for very rare diseases. Health Aff (Millwood). 2012;31(11):2528-35. DoI: https://doi.org/10.1377/ hlthaff.2012.0235.

31. Westley E, Schwarz EB. Emergency contraception: Global challenges, new opportunities. Contraception. 2012;85(5):429 31. DoI: https://doi.org/10.1016/j.contraception.2012.01.012.

32. Chaves GC, Hasenclever L, Osorio-de-Castro CG, et al. Strategies for price reduction of HIV medicines under a monopoly situation in Brazil. Rev Saúde Pública [internet]; 2015 [citado 2017 ago. 25]; 49(0):1-10. Disponible en: http://www.scielo.br/scielo. php?script=sci_arttext\&pid=S0034-89102015000100309\&lng $=$ en\&tlng=en.

33. Congreso de la República de Colombia. Ley 1753 de 2015. Plan Nacional de Desarrollo (2015 jun. 9).

34. World Health Organization (wHO). Essential medicines [internet]; s. f. [citado 2017 mar. 7]. Disponible en: http://www. who.int/medicines/services/essmedicines_def/en/.

35. Colombia, Ministerio de Salud y Protección Social Resolución 1692, por la cual se establecen los criterios para la compra centralizada, distribución y suministro de medicamentos para la hepatitis $\mathrm{C}$ crónica y el seguimiento a los pacientes diagnosticados con dicha patología y se dictan otras disposiciones (2017 may. 23)
36. Colombia, Ministerio de Salud y Protección Social. Medicamentos contra hepatitis C, ahora $80 \%$ más baratos [internet]; 2017 [citado 2018 feb. 13]. Disponible en: https:// www.minsalud.gov.co/Paginas/Medicamentos-contra-hepatitisC-ahora-80-mas-baratos.aspx.

37. Consultor Salud. Así quedaría control de precio de medicamentos [internet]; 2013 [citado 2018 abr. 23]. Disponible en: http:// www.consultorsalud.com/asi-quedaria-control-de-precio-demedicamentos

38. World Health Organization. Acuerdo sobre los aspectos de los derechos de propiedad intelectual relacionados con el comercio [internet]; 1995 [citado 2017 feb. 14]. Disponible en: https:// www.wto.org/SPANISH/DOCS_S/legal_s/27-trips.pdf.

39. Comisión de la Comunidad Andina. Decisión 486 de 2000 (2000 sep. 14).

40. Colombia, Ministerio de Salud y Protección Social. Resolución 2475 , por medio de la cual se adelanta una declaratoria de existencia de razones de interés público (2016 jun. 14).

41. Colombia, Ministerio de Salud y Protección Social, Dirección de Prestación de Servicios y Atención Primaria. Línea base de telemedicina en municipios priorizados - Colombia. Resultados generales [internet]; 2015 [citado 2018 may. 4]. Disponible en: https://www.minsalud.gov.co/sites/rid/Lists/ BibliotecaDigital/RIDE/VS/PSA/linea-base-telemedicinamunicpios-priorizados.pdf.

42. Colombia, Ministerio de Salud y Protección Social. Política de Atención Integral en Salud. Bogotá, Colombia; 2016. Disponible en: https://www.minsalud.gov.co/sites/rid/Lists/ BibliotecaDigital/RIDE/DE/modelo-pais-2016.pdf. 